\title{
A reverse KAM method to estimate unknown mutual inclinations in exoplanetary systems*
}

\author{
MARA VOLPI ${ }^{\dagger}$ \\ NaXys, Department of Mathematics, University of Namur \\ Rue Rempart de la Vierge 8, 5000 — Namur, Belgium. \\ UGO LOCATELLI \\ Dipartimento di Matematica, Università degli Studi di Roma "Tor Vergata", \\ via della Ricerca Scientifica 1, 00133 - Roma, Italy. \\ MARCO SANSOTTERA \\ Dipartimento di Matematica, Università degli Studi di Milano, \\ via Saldini 50, 20133 - Milano, Italy. \\ e-mails: mara.volpi@unamur.be, locatell@mat.uniroma2.it, \\ marco.sansottera@unimi.it
}

\begin{abstract}
The inclinations of exoplanets detected via radial velocity method are essentially unknown. We aim to provide estimations of the ranges of mutual inclinations that are compatible with the long-term stability of the system. Focusing on the skeleton of an extrasolar system, i.e., considering only the two most massive planets, we study the Hamiltonian of the three-body problem after the reduction of the angular momentum. Such a Hamiltonian is expanded both in Poincaré canonical variables and in the small parameter $D_{2}$, which represents the normalised Angular Momentum Deficit. The value of the mutual inclination is deduced from $D_{2}$ and, thanks to the use of interval arithmetic, we are able to consider open sets of initial conditions instead of single values. Looking at the convergence radius of the Kolmogorov normal form, we develop a reverse KAM approach in order to estimate the ranges of mutual inclinations that are compatible with the long-term stability in a KAM sense. Our method is successfully applied to the extrasolar systems HD 141399, HD 143761 and HD 40307.
\end{abstract}

\footnotetext{
${ }^{*}$ Key words and phrases: exoplanets, n-body planetary problem, KAM theory, Celestial Mechanics. 2010 Mathematics Subject Classification. Primary: 70F15; Secondary: 70H08, 37N05, 85A04, 85-08.

${ }^{\dagger}$ FRIA Research Fellow.
} 


\section{Introduction}

Nowadays, the number of catalogued multiple-planet systems is rapidly reaching one thousand. Their orbital characteristics are often quite different with respect to those of our Solar System (for a review containing a classification of the possible architectures see, e.g., 36]). In the present work, we will focus on the analysis of the long-term evolution of the observed exoplanets, in a spirit similar to the classical studies of stability of our Solar System.

Multiplanetary extrasolar systems raise new interesting challenges concerning the mathematical aspects of the orbital dynamics. For instance, in our Solar System the eccentricities of the celestial bodies play the role of small parameters in the power series expansions considered in classical perturbation theory. On the other hand, the observed eccentricities of the major planets in extrasolar systems are often so large (see, e.g., [4]), that they prevent the convergence of the Laplacian expansion of the disturbing function (see, e.g., 8]). Nevertheless, accurate analytical results based on classical expansions have been obtained even for systems having moderate eccentricities via high-order expansions (see, e.g., [23]).

In the present work, we limit our study to the exoplanets observed via radial velocity (hereafter, RV) method, because of its ability to detect massive bodies. Therefore, RV based observations are expected to capture information about the skeleton of an extrasolar system, i.e., its major planets. As a main drawback, the RV method cannot detect the orbital inclinations; moreover, its measure of the mass of each planet is affected by the uncertainty factor $\sin i$, being $i$ the inclination of the plane of motion with respect to the tangent plane to the celestial sphere (see, e.g., [2]). However, ranges of the most probable values of the inclinations can be deduced by prescribing the long-time stability of the system. This is done for instance in [19], where the properties of the numerically computed orbital motions are investigated by using the frequency analysis method (see 18 and references therein for an introduction to this kind of numerical explorations). We propose here a novel procedure: a reverse KAM approach by using normal forms depending on a free parameter related to the unknown mutual inclinations of the exoplanets. Our approach is based on a careful adaptation of the algorithm constructing the Kolmogorov normal form for the secular part of the Sun-Jupiter-Saturn (SJS) system (see [26]; see also [13, 1, 29], that are the original articles giving the name to the KAM theorem). The differences between the two contexts are remarkable. In [26] the parameters and the orbital elements of the SJS system were very well known; all these data were used to prove the existence of KAM tori confining the motion and, therefore, the stability of the secular model. Here, we deal with systems for which some of the orbital elements are unknown: we aim to infer information about their values by prescribing the stability and therefore requiring that the algorithm constructing KAM tori is convergent. Actually, from a practical point of view, its implementation is rather delicate. For instance, we use the interval arithmetic to represent the coefficients of the secular expansions; this allows us to consider sets of values of the free parameter in a comprehensive way instead of studying many different numerical integrations, each corresponding to a single value of that same parameter ranging in a suitably chosen discrete grid. Thus, our implementation 
is an interesting example of alternative use of validated numerics outside the context of a rigorous proof where it is often used (see, e.g., [7]). We emphasise that this is done by handling the difficulty due to the fact that the free parameter, related to the unknown mutual inclination, directly contributes to the so-called Laplace-Lagrange approximation (see, e.g., [23]). Therefore, it affects the secular frequencies possibly introducing dangerous resonance relations.

We believe that our approach can interestingly complement some recent results: in particular, the concept of "AMD-stability" introduced in [21] to analyse the dynamics of the multiple-planet extrasolar systems (see also [33] for an extension to the resonant case). Roughly, that criterion requires that the Angular Momentum Deficit 1 (hereafter AMD) is smaller than a critical threshold, in order to ensure that the planetary orbits cannot collide; therefore, the system is considered to be AMD-stable. In [21] five planetary systems are recognised to belong to the so-called subcategory of "hierarchical AMD-stable systems that are AMD-unstable but become AMD-stable when they are split into two parts". Among them, our Solar System is a typical example when considering the two subsystems formed by the giant planets on one side and the inner ones on the other. We emphasise that AMD-stability of the giant planets is not sufficient to prove the global stability of the system as it does not provide a detailed enough information about the regularity of their motions. Indeed, it is well known that the chaoticity of the secular motions of the inner planets is induced by the gravitational perturbations due to Jupiter (see [16]). Because of this chaoticity, it has been possible to select some scenarios (depicted by suitably chosen numerical integrations) leading to the ejection of Mercury or to destructive collisions between the terrestrial planets in a few billions of years (see [15] in the context of the secular dynamics and [20], respectively). It is very natural to expect that these destabilising effects would act dramatically faster, if also the secular dynamics of the outer system were chaotic, instead of being extremely regular as it has shown to be (see, e.g., [17] also as a review covering most of the properties of the Solar System that have been briefly recalled here). A deeper knowledge of the dynamics of the outer planets is therefore crucial in order to prove the effective (or long-time) stability of the complete system. When a specific extrasolar system cannot be classified as globally AMD-stable, the problem of ensuring its stability properties can be attacked by following a strategy that is based on our reverse KAM approach, as outlined below.

In the case of hierarchical AMD-stable systems, when successful our approach can ensure that there are values of the inclinations for which the subsystem formed by the major planets is stable in a much stronger sense with respect to the AMD-criterion: the eventual diffusion would be so weak that the orbit could not significantly go away from a KAM torus before an extremely 2 long interval of time (see [11]). In such a situation, the motion of the biggest planets is indistinguishable from a quasi-periodic one. Such

\footnotetext{
${ }^{1}$ The Angular Momentum Deficit is defined as the difference between the total value of the angular momentum and its value in the case of Keplerian circular coplanar orbits having radii equal to the semi-major axes of the planets.

2 Actually, when the mild hypotheses assumed in [28] are satisfied, the diffusion time is estimated to be super-exponentially big. This means that its order of magnitude is given by the exponential of the exponential of the inverse of a fractional power of the distance from a reference KAM torus.
} 
a preliminary result would be essential in order to prove (at least) the metastability of the less massive planets over times that are comparable with the expected lifetime of the system. This highlights the usefulness of our reverse KAM approach.

In the present paper, we apply our KAM-stability to three extrasolar systems that are modeled by a three-body approximation, which includes the star and the two biggest planets. Quite remarkably, one of these systems, HD 141399, is hierarchical AMD-stable according to the classifications given in [21]; another one, HD 40307, is included in the category of the AMD-unstable systems. This work represents the first step in the direction of a complete proof of the so-called effective stability of such exoplanetary systems, when they are studied in the framework of models including all their already discovered planets (see [35] for a recent application of these concepts to the secular dynamics of the Sun-Jupiter-Saturn-Uranus system). Of course, the whole implementation of our strategy is not priceless: the required amount of computations (mainly by the algebraic manipulations of the expansions) is extremely demanding.

Our paper is organised as follows. In Sect. 2 we recall the initial expansions of the secular Hamiltonian model of the three-body planetary problem. In Sect. 3 we deal with the algorithm constructing invariant tori for such a model. In Sect. 4 we describe the way to infer information about the range of values of the mutual inclination. The applications of our method to three extrasolar systems are discussed in Sect. 5. Finally, the conclusions are outlined in Sect. 6.

\section{Settings for the definition of the Hamiltonian model}

As it has been mentioned in the Introduction our approach is based on a careful adaptation of that described in [26]. However, for the sake of completeness, it is convenient to briefly recall both the definitions and the preliminary canonical transformations that properly introduce the secular model we are going to study.

\subsection{The expansion of the Hamiltonian}

Object of this study is a three-body planetary problem, formed by a central star (indicated by the index 0) and two planets revolving around it, marked by the indexes 1 (inner) and 2 (outer). Thanks to the conservation of the angular momentum, we can perform the reduction of the nodes which allows us to use the planar Poincaré variables

$$
\begin{array}{ll}
\Lambda_{j}=\frac{m_{0} m_{j}}{m_{0}+m_{j}} \sqrt{\left(m_{0}+m_{j}\right) a_{j}} \quad \lambda_{j}=M_{j}+\omega_{j} \\
\xi_{j}=\sqrt{2 \Lambda_{j}} \sqrt{1-\sqrt{1-e_{j}^{2}}} \cos \left(\omega_{j}\right) & \eta_{j}=-\sqrt{2 \Lambda_{j}} \sqrt{1-\sqrt{1-e_{j}^{2}}} \sin \left(\omega_{j}\right)
\end{array}
$$

where $a_{j}, e_{j}, M_{j}$ and $\omega_{j}$ are the semi-major axis, the eccentricity, the mean anomaly and perihelion argument of the $j$-th planet, respectively. We introduce the translation $L_{j}=\Lambda_{j}-\Lambda_{j}^{*}$, where $\Lambda_{j}^{*}$ is the value of $\Lambda_{j}$ for the observed semi-major axis $a_{j}$. 
Following [34, we expand the Hamiltonian both in the Poincaré variables and in the parameter $D_{2}$, that is a sort of "normalised angular momentum deficit",

$$
D_{2}=\frac{\left(\Lambda_{1}+\Lambda_{2}\right)^{2}-C^{2}}{\Lambda_{1} \Lambda_{2}}
$$

where $C$ is the norm of the total angular momentum: by definition, $D_{2}$ is $\mathcal{O}\left(e^{2}+i^{2}\right)$. This parameter is therefore a measure of the difference between the actual total angular momentum and the one of a similar system having circular and co-planar orbits (for which $\left.D_{2}=0\right)$. As a main difference with respect to the approach in [26], which we constantly refer to, here we keep $D_{2}$ as a free parameter in the expansions, while there it was replaced by its particular value (computed for the Sun-Jupiter-Saturn system). The Hamiltonian expansion in power series of the variables $\mathbf{L}, \boldsymbol{\xi}, \boldsymbol{\eta}$, parameter $D_{2}$ and in Fourier series of $\lambda$ writes

$$
H^{\left(\mathcal{T}_{F}\right)}=\sum_{j_{1}=1}^{\infty} h_{j_{1}, 0}^{(\mathrm{Kep})}(\mathbf{L})+\mu \sum_{s=0}^{\infty} \sum_{j_{1}=0}^{\infty} \sum_{j_{2}=0}^{\infty} D_{2}^{s} h_{s ; j_{1}, j_{2}}^{\left(\mathcal{T}_{F}\right)}(\mathbf{L}, \boldsymbol{\lambda}, \boldsymbol{\xi}, \boldsymbol{\eta})
$$

where $\mu=\max \left\{m_{1} / m_{0}, m_{2} / m_{0}\right\}$ and

- $h_{j_{1}, 0}^{(\mathrm{Kep})}$ is a homogeneous polynomial function (hereafter h.p.f.) of degree $j_{1}$ in $\mathbf{L}$; in particular, $h_{1,0}^{(\mathrm{Kep})}=\boldsymbol{n}^{*} \cdot \mathbf{L}$, where the components of the angular velocity vector $\boldsymbol{n}^{*}$ are defined by the third Kepler law.

- $h_{s ; j_{1}, j_{2}}^{\left(\mathcal{T}_{F}\right)}$ is a h.p.f. of degree $j_{1}$ in $\mathbf{L}$, degree $j_{2}$ in $\boldsymbol{\xi}$ and $\boldsymbol{\eta}$, and with coefficients that are trigonometric polynomials in $\boldsymbol{\lambda}$.

\subsection{The secular Hamiltonian at order two in the masses}

The main idea of this work is based on the construction of invariant tori through the application of a Kolmogorov normalisation algorithm. The Kolmogorov normalisation scheme is also adapted to preliminary produce the secular approximation at order two in the masses (see, e.g., [26, 23]). This means that in our model the torus corresponding to $\mathbf{L}=0$ in the new coordinates will be invariant up to order two in the masses. For this aim, we proceed by averaging over the fast angles the terms of the Hamiltonian (3) that do not depend or are linear in the actions $\mathbf{L}$. This elimination is obtained via a composition of two Kolmogorov-like steps.

First, the transformed Hamiltonian writes, in the Lie series formalism,

$$
\exp \mathcal{L}_{\chi_{1}^{\left(\mathcal{O}_{2}\right)}} H^{\left(\mathcal{T}_{F}\right)}=\sum_{j=0}^{\infty} \frac{1}{j !} \mathcal{L}_{\chi_{1}^{j}}^{j(\mathcal{O} 2)} H^{\left(\mathcal{T}_{F}\right)}
$$

where the generating function $\chi_{1}^{(\mathcal{O} 2)}$ is determined as the solution of the following homological equation

$$
\sum_{i=1}^{2} n_{i}^{*} \cdot \frac{\partial \chi_{1}^{(\mathcal{O} 2)}}{\partial \lambda_{i}}+\mu \sum_{\substack{s=0, j_{2}=0 \\ 2 s+j_{2} \leq N_{S}}}\left[D_{2}^{s} h_{s ; 0, j_{2}}^{\left(\mathcal{T}_{F}\right)}\right]_{\boldsymbol{\lambda}: K_{F}}=\mu \sum_{\substack{s=0, j_{2}=0 \\ 2 s+j_{2} \leq N_{S}}} D_{2}^{s}\left\langle h_{s ; 0, j_{2}}^{\left(\mathcal{T}_{F}\right)}\right\rangle_{\boldsymbol{\lambda}},
$$


being $\langle\cdot\rangle_{\varphi}$ the average over the generic angles $\varphi$. In the previous formula, we have denoted with $\lceil g\rceil_{\lambda: K_{F}}$ the truncation of the expansion of the generic function $g$ up to a trigonometric degree $K_{F}$. The parameter $K_{F}$ is fixed so as to include the main quasiresonance of the system on hand: for instance, let us suppose the system is near to a $k_{1}^{*}: k_{2}^{*}$ resonance, then we set $K_{F} \geq\left|k_{1}^{*}\right|+\left|k_{2}^{*}\right|$. Moreover, in (5) the integer parameter $N_{S}$ rules the considered order of magnitude in eccentricity and inclination: the choice of the particular value of $N_{S}$ is again related to the main quasi-resonance of the system. In fact, for the D'Alembert rules we know that the terms containing harmonics $\left(k_{1}^{*} \lambda_{1}-k_{2}^{*} \lambda_{2}\right)$ have order in eccentricity and inclination greater or equal than $\left|k_{1}^{*}\right|-\left|k_{2}^{*}\right|$ and with the same parity. Therefore, in order to include the effects of the $k_{1}^{*}: k_{2}^{*}$ resonance in the generating function $\chi_{1}^{(\mathcal{O} 2)}$, we have to truncate the expansion up to $N_{S} \geq\left|k_{1}^{*}\right|-\left|k_{2}^{*}\right|$. This constraint takes into account that both $\boldsymbol{\xi}$ and $\boldsymbol{\eta}$ are $\mathcal{O}(e)$ and $D_{2}$ is $\mathcal{O}\left(e^{2}+i^{2}\right)$. To fix the ideas, let us focus on the main extrasolar planets HD 141399 c and HD 141399 d, whose periods are approximately equal to 202 days and 1070 days, respectively. Therefore, the quasi-resonance 5:1 is expected to substantially affect the dynamics: according to the previous discussion, we then fix $K_{F} \geq 6$ and $N_{S} \geq 4$. Of course, these criteria determine just the lower bounds on the integer parameters $K_{F}$ and $N_{s}$ : we stress that one could be interested in producing larger expansions according to the available computational power. In Section 5 , Tab. 2 we will list the particular value of $K_{F}$ and $N_{S}$ for each of the systems considered by our applications.

The second Kolmogorov-like step is performed in an analogous way so as to introduce the normalised Hamiltonian up to order two in the masses $H^{\left(\mathcal{O}_{2}\right)}=\exp \mathcal{L}_{\chi_{2}^{\left(\mathrm{O}_{2}\right)}}$ 。 $\exp \mathcal{L}_{\chi_{1}^{\left(\mathcal{O}_{2}\right)}} H^{\left(\mathcal{T}_{F}\right)}$, where the new generating function $\chi_{2}^{\left(\mathrm{O}_{2}\right)}$ is the solution of the homological equation

$$
\sum_{i=1}^{2} n_{i}^{*} \cdot \frac{\partial \chi_{2}^{(\mathcal{O} 2)}}{\partial \lambda_{i}}+\mu \sum_{\substack{s=0, j_{2}=0 \\ 2 s+j_{2} \leq N_{S}}}\left\lceil D_{2}^{s} h_{s ; 1, j_{2}}^{\left(\mathcal{T}_{F}\right)}\right]_{\lambda: K_{F}}+\mathcal{L}_{\chi_{1}^{(\mathcal{O} 2)}} h_{2,0}^{(\mathrm{Kep})}=\mu \sum_{\substack{s=0, j_{2}=0 \\ 2 s+j_{2} \leq N_{S}}} D_{2}^{s}\left\langle h_{s ; 1, j_{2}}^{\left(\mathcal{T}_{F}\right)}\right\rangle_{\lambda} .
$$

As we already mentioned, we will focus on the secular part of the Hamiltonian $\left\langle H^{(\mathcal{O} 2)}\right\rangle_{\lambda}$ : for such an Hamiltonian, the actions $\mathbf{L}$ are first integrals. We consider the basic approximation of the fast dynamics corresponding to quasi-periodic motions with an angular velocity vector equal to $\boldsymbol{n}^{*}$, by setting $\mathbf{L}=0$.

Let us define

$$
\begin{aligned}
\widetilde{H} & =H^{\left(\mathcal{T}_{F}\right)}+\frac{1}{2}\left\{\chi_{1}^{(\mathcal{O} 2)}, \mathcal{L}_{\chi_{1}^{(\mathcal{O} 2)}} h_{2,0}^{(\mathrm{Kep})}\right\}_{\boldsymbol{L}, \boldsymbol{\lambda}} \\
& +\left\{\chi_{1}^{(\mathcal{O} 2)}, \mu \sum_{\substack{s=0, j_{2}=0 \\
2 s+j_{2} \leq N_{S}}} D_{2}^{s} \tilde{h}_{s ; 1, j_{2}}^{\left(\mathcal{T}_{F}\right)}\right\}_{\boldsymbol{L}, \boldsymbol{\lambda}}+\frac{1}{2}\left\{\chi_{1}^{(\mathcal{O} 2)}, \mu \sum_{\substack{s=0, j_{2}=0 \\
2 s+j_{2} \leq N_{S}}} D_{2}^{s} \tilde{h}_{s ; 0, j_{2}}^{\left(\mathcal{T}_{F}\right)}\right\}_{\boldsymbol{\xi}, \boldsymbol{\eta}}
\end{aligned}
$$

where $\{\cdot, \cdot\}_{\boldsymbol{L}, \boldsymbol{\lambda}}$ and $\{\cdot, \cdot\}_{\boldsymbol{\xi}, \boldsymbol{\eta}}$ are the terms of the Poisson bracket involving only the derivatives with respect to the variables $(L, \lambda)$ and $(\xi, \eta)$, respectively. Then, according to [26], we have that

$$
\left.\left\langle H^{(\mathcal{O} 2)}\right\rangle_{\boldsymbol{\lambda}}\right|_{\mathbf{L}=\mathbf{0}}=\left.\langle\widetilde{H}\rangle_{\boldsymbol{\lambda}}\right|_{\mathbf{L}=\mathbf{0}}+\mathcal{O}\left(\mu^{3}\right)
$$


We can finally introduce our secular model up to order two in the masses, by setting

$$
H^{(\mathrm{sec})}\left(D_{2}, \boldsymbol{\xi}, \boldsymbol{\eta}\right)=\left\lceil\left.\langle\widetilde{H}\rangle_{\boldsymbol{\lambda}}\right|_{\mathbf{L}=\mathbf{0}}\right\rceil_{\left(D_{2}, \boldsymbol{\xi}, \boldsymbol{\eta}\right): 2 N_{S}},
$$

where $\left\lceil\left.\langle\widetilde{H}\rangle_{\boldsymbol{\lambda}}\right|_{\mathbf{L}=\mathbf{0}}\right\rceil_{\left(D_{2}, \boldsymbol{\xi}, \boldsymbol{\eta}\right): 2 N_{S}}$ indicates the averaged expansion (over the fast angles $\boldsymbol{\lambda}$ ) of the part of $\widetilde{H}$ that is both independent from the actions $\mathbf{L}$ and truncated up to a total order of magnitude $N_{S}$ in eccentricity and inclination. This means that a monomial $D_{2}^{s} \boldsymbol{\xi}^{\boldsymbol{m}_{1}} \boldsymbol{\eta}^{\boldsymbol{m}_{2}}$ is included in the truncation if and only if $2 s+\left|\boldsymbol{m}_{1}\right|+\left|\boldsymbol{m}_{2}\right| \leq 2 N_{S}$. By comparing (77) and (8), one can notice that our secular Hamiltonian model represented by $H^{(\mathrm{sec})}$ does not depend on the second generating function $\chi_{2}^{(\mathrm{O} 2)}$ whose explicit calculation is therefore unnecessary.

The explicit form of (8) writes

$$
H^{(\mathrm{sec})}=h_{1,1}^{(\mathrm{sec})}+\sum_{s=2}^{N_{S}} \sum_{l=1}^{s} D_{2}^{s-l} h_{s, l}^{(\mathrm{sec})}
$$

where $h_{s, l}$ is a homogeneous polynomial function of degree $2 l$ in $\boldsymbol{\xi}$ and $\boldsymbol{\eta}, \forall 1 \leq l \leq s \leq$ $N_{S}$. The even parity of the exponents is determined by the D'Alembert rules: having removed all the harmonics, the order in eccentricity that the terms must held is of the same parity of zero. The expansion of the final Hamiltonian $H^{(\mathrm{sec})}$ presents terms in $D_{2}$, $\boldsymbol{\xi}$ and $\boldsymbol{\eta}$ up to a degree that is twice the one of the truncated expansions of $\chi_{1}^{(\mathrm{O} 2)}$ as it is determined by (5): this is set to ensure that all the terms generated by the Poisson brackets in (7) are going to be taken into account.

\section{Construction of invariant tori for our secular model}

\subsection{Preliminary set up for the Kolmogorov algorithm}

We will perform a series of preliminary transformations in order to obtain the most convenient formulation of our Hamiltonian for the construction of the invariant torus. Firstly, we will diagonalise the quadratic part of the Hamiltonian; secondly, we will transform the variables into an action-angle set; we will then proceed with a partial Birkhoff's normalisation, so as to remove the degeneration of the unperturbed Hamiltonian; finally, we will shift the origin of the actions so that they are centred around a value consistent with the observations.

It is well known that under mild assumptions on the quadratic part of the Hamiltonian which are satisfied in our case (see Sect. 3 of [3] where such hypotheses are shown to be generically fulfilled for a planar model of our Solar System) one can find a canonical transformation $(\boldsymbol{\xi}, \boldsymbol{\eta})=\mathcal{D}(\boldsymbol{x}, \boldsymbol{y})$ with the following properties: (i) the map $(\boldsymbol{\xi}, \boldsymbol{\eta})=(\boldsymbol{\xi}(\boldsymbol{x}), \boldsymbol{\eta}(\boldsymbol{y}))$ is linear, (ii) $\mathcal{D}$ diagonalizes the quadratic part of the Hamiltonian, so that we can write $h_{1,1}^{\text {(sec) }}$ in the new coordinates as $\sum_{j=1}^{2} \nu_{j}\left(x_{j}^{2}+y_{j}^{2}\right) / 2$, where both the entries of the vector $\boldsymbol{\nu}$ have the same sign. 
Action-angle variables are introduced via the canonical transformation

$$
x_{j}=\sqrt{2 I_{j}} \cos \varphi_{j}, \quad y_{j}=\sqrt{2 I_{j}} \sin \varphi_{j}, \quad j=1,2 .
$$

With these two last changes of coordinates the Hamiltonian (9) takes the form

$$
H^{(\mathrm{I})}(\boldsymbol{I}, \boldsymbol{\varphi})=\boldsymbol{\nu} \cdot \boldsymbol{I}+\sum_{s=2}^{\infty} \sum_{l=1}^{s} D_{2}^{s-l} h_{s ; l}^{(\mathrm{I})}(\boldsymbol{I}, \boldsymbol{\varphi})
$$

where $h_{s ; l}$ is an homogeneous polynomial function of degree $2 l$ in the square roots of actions $\boldsymbol{I}$ and a trigonometric polynomial of degree $2 s$ in angles $\boldsymbol{\varphi}$, i.e., it writes

$$
h_{s, l}^{(\mathrm{I})}(\boldsymbol{I}, \boldsymbol{\varphi})=\sum_{i_{1}+i_{2}=2 l} \sum_{j_{1}=0}^{i_{1}} \sum_{j_{2}=0}^{i_{2}} c_{s ; i_{1} ; i_{2} ; j_{1} ; j_{2}}^{(\mathrm{I})} \sqrt{I_{1}^{i_{1}} I_{2}^{i_{2}}} \cos \left[\left(i_{1}-2 j_{1}\right) \varphi_{1}+\left(i_{2}-2 j_{2}\right) \varphi_{2}\right] .
$$

In the previous formula only cosines occur because of the parity relation due to the D'Alembert rules.

Let us stress that our aim is to provide ranges of inclinations which are compatible with the stability of the system. These intervals of values are obtained as a function of the angular momentum deficit parameter $D_{2}$. Thus it is crucial to keep $D_{2}$ as a parameter in the Hamiltonian expansion as long as possible. We now proceed with a partial Birkhoff's normalisation in order to remove the degeneration of the unperturbed Hamiltonian. We can visualise the Hamiltonian (11) as

$$
\begin{aligned}
& h_{4 ; 4}^{(\mathrm{I})} \quad \ldots \\
& h_{3 ; 3}^{(\mathrm{I})} \quad D_{2} h_{4 ; 3}^{(\mathrm{I})} \quad \ldots \\
& h_{2 ; 2}^{(\mathrm{I})} \quad D_{2} h_{3 ; 2}^{(\mathrm{I})} \quad D_{2}^{2} h_{4 ; 2}^{(\mathrm{I})} \quad \ldots \\
& H^{(\mathrm{I})}(\boldsymbol{I}, \boldsymbol{\varphi})=\boldsymbol{\nu} \cdot \boldsymbol{I} \quad D_{2} h_{2 ; 1}^{(\mathrm{I})} \quad D_{2}^{2} h_{3 ; 1}^{(\mathrm{I})} \quad D_{2}^{3} h_{4 ; 1}^{(\mathrm{I})} \quad \ldots .
\end{aligned}
$$

This writing highlights two features of each term: the size of the perturbation in eccentricity and inclination is determined by the columns; the degree in actions depends on the rows. Our aim is then to remove the dependency on the angle variables up to the third column. We determine the first generating function by solving

$$
\left\{\mathcal{B}_{1}^{(\mathrm{II})}, \boldsymbol{\nu} \cdot \boldsymbol{I}\right\}-D_{2} h_{2 ; 1}^{(\mathrm{I})}=D_{2} Z_{2 ; 1}
$$

where $Z_{s, l}$ is the average of $h_{s ; l}^{(\mathrm{I})}$ over the angles $\boldsymbol{\varphi}$.

In the same way, we determine the generating function $\mathcal{B}_{2}^{(\mathrm{II})}$ as the solution of

$$
\left\{\mathcal{B}_{2}^{(\mathrm{II})}, \boldsymbol{\nu} \cdot \boldsymbol{I}\right\}-h_{2 ; 2}^{(\mathrm{I})}=Z_{2 ; 2} .
$$

The transformed Hamiltonian is computed as

$$
H^{(\mathrm{II})}=\exp \mathcal{L}_{\mathcal{B}_{2}^{(\mathrm{II})}} \circ \exp \mathcal{L}_{\mathcal{B}_{1}^{(\mathrm{II})}} H^{(\mathrm{I})} .
$$


Let us stress that $\exp \mathcal{L}_{\mathcal{B}_{1}^{(\mathrm{II})}} H^{(\mathrm{I})}$ does not produce any contribution to the term $h_{2 ; 2}^{(\mathrm{I})}$ : this justifies the term appearing in (15) for the generating function. At this point, all the terms up to order 4 in eccentricity and inclination do not depend on the fast angles and the Hamiltonian reads

$$
H^{(\mathrm{II})}(\boldsymbol{I}, \boldsymbol{\varphi})=\boldsymbol{\nu} \cdot \boldsymbol{I}+D_{2} Z_{2 ; 1}(\boldsymbol{I})+Z_{2 ; 2}(\boldsymbol{I})+\sum_{s=3}^{\infty} \sum_{l=1}^{s} D_{2}^{s-l} h_{s ; l}^{(\mathrm{II})}(\boldsymbol{I}, \boldsymbol{\varphi}) .
$$

Analogously, we compute the generating functions $\mathcal{B}_{1}^{(\mathrm{III})}, \mathcal{B}_{2}^{(\mathrm{III})}, \mathcal{B}_{3}^{(\mathrm{III})}$ in order to eliminate the dependency on the angle variables of the terms of order 6 in eccentricity and inclinations. Finally, our Hamiltonian is computed as

$$
H^{(\mathrm{III})}=\exp \mathcal{L}_{\mathcal{B}_{3}^{(\mathrm{III})}} \circ \exp \mathcal{L}_{\mathcal{B}_{2}^{(\mathrm{III})}} \circ \exp \mathcal{L}_{\mathcal{B}_{1}^{(\mathrm{III})}} H^{(\mathrm{II})} .
$$

The last preliminary transformation of the Hamiltonian consists in a translation of the actions. Being the action vector $\boldsymbol{I}$ nearly constant, i.e., $\boldsymbol{I}(t) \simeq \boldsymbol{I}(0)$, we shift the origin of the action about $\boldsymbol{I}(0)=\boldsymbol{I}^{*}$. This is done using a canonical transformation $\mathcal{T}(\boldsymbol{I}, \boldsymbol{\varphi})=\left(\boldsymbol{p}+\boldsymbol{I}^{*}, \boldsymbol{q}\right)$. The transformed Hamiltonian is given by

$$
H^{(0)}(\boldsymbol{p}, \boldsymbol{q})=H^{(\mathrm{III})} \circ \mathcal{T}(\boldsymbol{I}, \boldsymbol{\varphi}) .
$$

Let us remark that in [26] the translation was determined in such a way to construct a torus with a specific frequency $\boldsymbol{\omega}$; that frequency was accurately computed from the numerical integration via Fourier analysis. The same approach cannot be adopted in the present work: being the knowledge of the parameters of the system only partial any numerically integration is unattainable.

\subsection{Formal construction of the Kolmogorov invariant tori}

We will now proceed with the construction of the Kolmogorov invariant tori. Firstly, we expand the Hamiltonian $H^{(0)}$, whose expansion can be visually arranged as

$$
\begin{array}{cccccc} 
& \vdots & \vdots & \vdots & \vdots & \vdots \\
f_{2}^{(0,0)(\boldsymbol{p})} & f_{2}^{(0,1)}(\boldsymbol{p}, \boldsymbol{q}) & \ldots & f_{2}^{(0, s)}(\boldsymbol{p}, \boldsymbol{q}) & \ldots \\
& \boldsymbol{\omega}^{(0)} \cdot \boldsymbol{p} & f_{1}^{(0,1)}(\boldsymbol{p}, \boldsymbol{q}) & \ldots & f_{1}^{(0, s)}(\boldsymbol{p}, \boldsymbol{q}) & \ldots \\
0 & f_{0}^{(0,1)}(\boldsymbol{q}) & \ldots & f_{0}^{(0, s)}(\boldsymbol{q}) & \ldots
\end{array}
$$

being the generic term $f_{j}^{(0, s)} \in \mathcal{P}_{j, 2 s}$. This means that it is a homogeneous polynomial of degree $j$ in the actions $\boldsymbol{p}$ and a trigonometric polynomial of degree $2 s$ in $\boldsymbol{q}$. Therefore it is possible to represent such type of terms on a computer because it is finite. There is a striking difference between the visual schemes (13) and (19): in the latter, we do not keep track of the expansions in powers of $D_{2}$. This is due to the fact that, in the explicit applications, we replace the parameter $D_{2}$ with convenient intervals of values. 
In Sec. 4 we will discuss in more detail this technical point, that is not essential for the comprehension of the normalisation scheme.

Let us emphasise that the terms $f_{j}^{(0, s)}$ in the $s$-th column are of order $\left\|\boldsymbol{I}^{*}\right\|^{s}$, as it is discussed, e.g., in [11]. Therefore the parameter $\boldsymbol{I}^{*}$ rules the convergence of the series with respect to the index $s$; according to the definitions in the previous sections, it is a small quantity because $\boldsymbol{I}^{*}$ is $\mathcal{O}\left(e^{2}+i^{2}\right)$.

The Kolmogorov's normalisation algorithm requires to remove all the terms of the Hamiltonian (19) of degree 0 or 1 in the actions $\boldsymbol{p}$, with the exception of the term $\boldsymbol{\omega} \cdot \boldsymbol{p}$. In order to do that, we start by determining the generating function $\chi_{1}^{(1)}$ such that

$$
\left\{\chi_{1}^{(1)}, \boldsymbol{\omega}^{(0)} \cdot \boldsymbol{p}\right\}+f_{0}^{(0,1)}=0,
$$

where $\chi_{1}^{(1)}$ is a trigonometric polynomial of degree 2 .

We will then obtain a new Hamiltonian

$$
\hat{H}^{(1)}=\exp \mathcal{L}_{\chi_{1}^{(1)}} H^{(0)}
$$

whose generic term of the expansion is $\hat{f}_{j}^{(1, s)} \in \mathcal{P}_{j, 2 s}$. As a consequence of equation (20), we have that $\hat{f}_{0}^{(1,1)}=0$.

We proceed in an analogous way to complete this first Kolmogorov's normalisation step: we compute the generating function $\chi_{2}^{(1)}(\boldsymbol{p}, \boldsymbol{q})$ such that

$$
\left\{\chi_{2}^{(1)}, \boldsymbol{\omega}^{(0)} \cdot \boldsymbol{p}\right\}+\hat{f}_{1}^{(1,1)}=\left\langle\hat{f}_{1}^{(1,1)}\right\rangle_{\boldsymbol{q}}
$$

then, $\chi_{2}^{(1)}$ will be linear in $\boldsymbol{p}$ and of order 2 in $\boldsymbol{q}$. Let us stress that it is possible to solve the previous homological equations (20) and (22), provided that $\left|\boldsymbol{k} \cdot \boldsymbol{\omega}^{(0)}\right|>0$ for $\boldsymbol{k} \in \mathbb{Z}_{2}$ with $|\boldsymbol{k}|=1,2$, being $|\boldsymbol{k}|=\left|k_{1}\right|+\left|k_{2}\right|$.

Therefore, we will obtain the new Hamiltonian $H^{(1)}=\exp \mathcal{L}_{\chi_{2}^{(1)}} \hat{H}^{(1)}$, whose generic term is now $f_{j}^{(1, s)} \in \mathcal{P}_{j, 2 s}$. In the following it lies a profound difference with respect to previous works (see for example [26]): due to the way $\chi_{2}^{(1)}$ was determined, we have that

$$
f_{1}^{(1,1)}=\hat{f}_{1}^{(1,1)}+\mathcal{L}_{\chi_{2}^{(1)}} \boldsymbol{\omega}^{(0)} \cdot \boldsymbol{p}=\left\langle\hat{f}_{1}^{(1,1)}\right\rangle_{\boldsymbol{q}}
$$

Therefore, $f_{1}^{(1,1)}$ is an homogeneous polynomial of degree 1 in $\boldsymbol{p}$ and independent from $\boldsymbol{q}$ : hence, it shares the same functional properties of the term $\boldsymbol{\omega}^{(0)} \cdot \boldsymbol{p}$. We then set for appropriate values of $\boldsymbol{\omega}^{(1)}$

$$
\boldsymbol{\omega}^{(1)} \cdot \boldsymbol{p}=\boldsymbol{\omega}^{(0)} \cdot \boldsymbol{p}+\left\langle\hat{f}_{1}^{(1,1)}\right\rangle_{\boldsymbol{q}}
$$

hence changing the frequency vector associated to the searched invariant tori.

The generic $r$-th normalisation step is performed in the same way provided that the following non-resonance condition holds true:

$$
\left|\boldsymbol{k} \cdot \boldsymbol{\omega}^{(r-1)}\right|>0, \quad \forall \boldsymbol{k} \in \mathbb{Z}_{2} \backslash\{\mathbf{0}\} \text { with }|\boldsymbol{k}| \leq 2 r .
$$


One can start from an expansion of the Hamiltonian $H^{(r-1)}$ of the same form as in (19), where the upper index 0 is replaced by $r-1$. Hence, the generating functions $\chi_{1}^{(r)}, \chi_{2}^{(r)}$ are introduced by solving the homological equations obtained by replacing the upper index 1 with $r$ in formulas (20) and (22).

The new Hamiltonian is therefore given by

$$
H^{(r)}=\exp \mathcal{L}_{\chi_{2}^{(r)}} \hat{H}^{(r)} \quad \text { with } \quad \hat{H}^{(r)}=\exp \mathcal{L}_{\chi_{1}^{(r)}} H^{(r-1)} .
$$

In order to better understand the ultimate goal of this algorithm constructing invariant tori, let us suppose to be able to iterate it ad infinitum. We would end up with a Hamiltonian of the form

$$
H^{(\infty)}(\boldsymbol{p}, \boldsymbol{q})=\boldsymbol{\omega}^{(\infty)} \cdot \boldsymbol{p}+\mathcal{O}\left(\|\boldsymbol{p}\|^{2}\right)
$$

Writing the equations of motion derived from the previous Hamiltonian, it appears evident that the torus $\left\{\boldsymbol{p}=\mathbf{0}, \boldsymbol{q} \in \mathbb{T}^{2}\right\}$ is invariant.

\section{Parametric study on the $D_{2}$ parameter}

By borrowing the techniques used in [10] to ensure the existence of elliptic tori for planetary systems, one could prove the convergence of the algorithm described in the previous section under very general conditions. In practice, this means that: (i) the perturbation (ruled by $\boldsymbol{I}^{*}$ ) is small enough; (ii) the hessian of the main quadratic term $f_{2}^{(0,0)}(\boldsymbol{p})$ is non-degenerate; (iii) the initial frequencies $\boldsymbol{\omega}^{(0)}$ belong to a suitable set having non-zero Lebesgue measure.

Here we do not investigate theoretically the convergence of the algorithm that is instead numerically analysed. In the spirit of a reverse KAM approach, we claim that some initial conditions originate motions that are inside a stable region when the convergence is evident from a numerical point of view.

We want to investigate the stability of extrasolar planetary systems for the widest possible ranges of $D_{2}$ (i.e., mutual inclinations) and we want to take into account the uncertainties on other orbital elements due to the observational limitations. Therefore, we have found convenient to represent the coefficients of the expansions of the Hamiltonians with intervals. Let us emphasise that such an approach based on interval arithmetic allows us to cover completely a set of values of the orbital elements. This provides a key advantage to the normal form approach with respect to the explorations purely based on numerical integrations. In fact, when dealing with numeric parametrical analysis the latter methods require to consider grids of values of the initial conditions; moreover, the synthetic coverage provided by the normal form approach (implemented with interval arithmetic) is not possible.

When dealing with the proof of any KAM-type statement, it is essential to establish an iterative scheme of estimates producing suitable majorants. The ultimate goal of such a scheme is proving that the norms of the two sequences of generating functions (i.e., $\chi_{1}^{(r)}$ and $\chi_{2}^{(r)}$ in our settings) decrease exponentially. Therefore, when such a behaviour 
is met in the plot of the norms of the generating functions, this is the clear signature of the existence of invariant KAM tori. In the case of a forced pendulum Hamiltonian model (see [7]), the study of the behaviour of $\chi_{2}^{(r)}$ succeeded in extrapolating a good approximation of the breakdown of the golden ratio invariant torus. As it has been discussed in the Introduction, the existence of KAM tori implies the long-time stability of the dynamics in a region surrounding them. Therefore, this argument ensures that the stability in KAM sense is firmly related to the convergence of the generating functions.
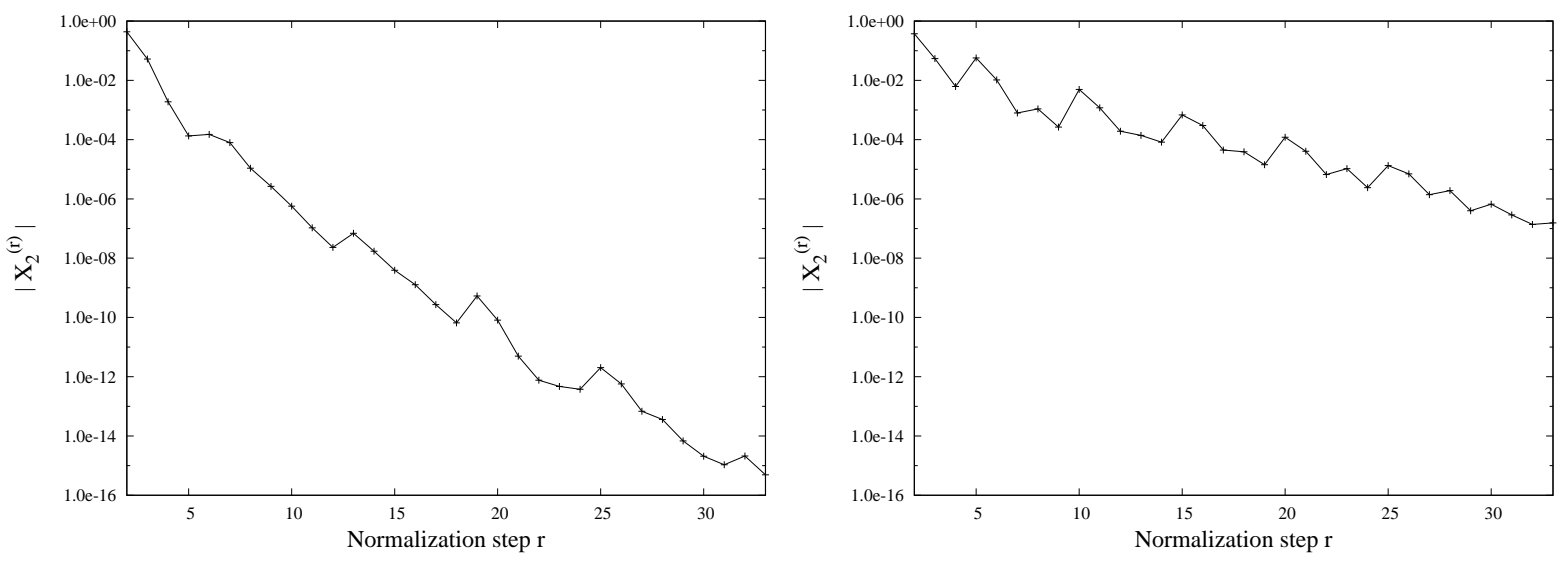

Figure 1: Results relative to HD 40307. Behaviour of the norms of the generating functions $\chi_{2}^{(r)}$ as a function of the normalisation step $r$. On the left, for values of $D_{2} \in[0.0164,0.0564]$. On the right for values of $D_{2} \in[0.0814,0.0864]$. The orbital parameters of the system are listed in Tab. 1.

To fix the ideas, let us consider the specific case of the extrasolar multiplanetary system HD 40307, whose orbital parameters are reported in Tab. 1. The plots of the norms of the generating functions $\chi_{2}^{(r)}$ are shown in Fig. 1 for two different ranges of values of the parameter $D_{2}$. The norm $\left\|\chi_{2}^{(r)}\right\|$ is nothing but the sum of the absolute values of the coefficients of the terms appearing in its expansion. We decide to focus on the behaviour of $\chi_{2}^{(r)}$ instead of the one of $\chi_{1}^{(r)}$ because the former ones are usually bigger than the latters. On the left hand side of Fig. 1 we can appreciate that the decrease of $\left\|\chi_{2}^{(r)}\right\|$ is sharp and quite regular; we associate this behaviour to the convergence of the algorithm. Often the algorithm crashes because the coefficients in the expansions of the Hamiltonians inflate to the point where the non-resonant condition (25) is not satisfied anymore. By comparison, the decrease of the norms in the plot on the right of Fig. 1 is notably slower than the one on the left; for instance, the norm of the last computed generating function on the right is 6 orders of magnitude bigger than the corresponding on the left.

Obviously we aim to automatise the identification of the converging procedures to avoid a visual inspection for each specific instance. Having fixed the maximal normalisation order at $\bar{r}=33$, in our codes the non convergence is established if at least one of the following tests is true:

1. the ratio $\left\|\chi_{2}^{(r)}\right\| /\left\|\chi_{2}^{(1)}\right\|$ is greater than $0.9^{r-1}$ for some $r$; 


\begin{tabular}{ccccccc}
\hline System & Planet & $\begin{array}{c}m \sin i \\
{\left[M_{J}\right]}\end{array}$ & $\begin{array}{c}M_{\text {Star }} \\
{\left[M_{\odot}\right]}\end{array}$ & $\begin{array}{c}a \\
{[\mathrm{AU}]}\end{array}$ & $e$ & $\begin{array}{c}\omega \\
{\left[{ }^{\circ}\right]}\end{array}$ \\
\hline HD 141399 & $c$ & 1.33 & 1.14 & 0.704 & $0.048 \pm 0.009$ & $220 \pm 40$ \\
& $d$ & 1.18 & & 2.14 & $0.074 \pm 0.025$ & $220 \pm 30$ \\
HD 143761 & $b$ & 1.045 & 0.99 & 0.228 & $0.037 \pm 0.004$ & $270.6 \pm 6$ \\
& $c$ & 0.079 & & 0.427 & $0.050 \pm 0.004$ & $175 \pm 125$ \\
HD 40307 & $c$ & 0.0202 & 0.77 & 0.081 & $0.060 \pm 0.005$ & $234 \pm 1$ \\
& $d$ & 0.0275 & & 0.134 & $0.070 \pm 0.005$ & $170 \pm 10$ \\
\hline
\end{tabular}

Table 1: Orbital parameters of the systems considered to apply the computational algorithm for the parametric study on $D_{2}$. For each column the unit of measure is reported in square brackets. The angle $i$ refers to the inclination of the orbital plane with respect to the line of sight.

2. the norm $\left\|\chi_{2}^{(\bar{r})}\right\|$ is greater than $10^{-9}\left\|\chi_{2}^{(1)}\right\|$.

Otherwise, we assume it is convergent.

\section{Results}

In order to explicitly apply our approach, we selected extrasolar systems where the eccentricities of the two major planets are small (i.e., less than 0.1). In Tab. 1 we report the orbital parameters of the systems considered: for the sake of simplicity in the following we use as planetary masses the minimal ones listed there.

For the sake of completeness, we define some of the parameters ruling the finite size of the expansions of the Hamiltonians introduced in our formal algorithm (Secs. 22 3). In Tab. 2 we list the values of the integer parameters $K_{F}$ and $N_{S}$ and of the mean motion resonance that is considered to play the major role in the perturbation of the non-resonant fast dynamics. Let us recall that $K_{F}$ gives the limitation on the generating function $\chi_{1}^{\left(\mathrm{O}_{2}\right)}$ that is needed to construct the approximation of order 2 in the masses; moreover, $N_{S}$ fixes the maximal order in $e^{2}+i^{2}$ for the secular Hamiltonian $H^{(\mathrm{sec})}$ (see Sec. 2.2). The series appearing in (11) and defining $H^{(\mathrm{I})}$ is truncated at the final value $s=15$; the same limitation is imposed on the expansions of $H^{(\mathrm{II})}$ and $H^{(\mathrm{III})}$. Finally, the maximal degree in the actions $\boldsymbol{p}$ is fixed at 4 for the expansions of all the Hamiltonians $H^{(r)}$ involved in the normalisation up to order $\bar{r}=33$.

In Fig. 2 we present two plots relative to HD 141399. On the left, we show the True/False output which results from the tests on the convergence described in Sec. 4, to each value of the parameter $D_{2}$ we assign 1 if the system is convergent, 0 otherwise. On the right, we show the plot of the the mutual inclination as a function of the parameter $D_{2}$. By means of the interval arithmetic, we can take into account the observational errors on the orbital parameters of the system, e.g., the eccentricities (as shown in Tab. 1). Therefore, for each value of the parameter $D_{2}$ we obtain a range of values for the mutual 


\begin{tabular}{cccc}
\hline System & Nearest Resonance & $K_{F}$ & $N_{S}$ \\
\hline HD 141399 & $5: 1$ & 12 & 8 \\
HD 143761 & $5: 2$ & 8 & 6 \\
HD 40307 & $2: 1$ & 6 & 8 \\
\hline
\end{tabular}

Table 2: Nearest resonance and values of the integer parameter $K_{F}$ and $N_{S}$ (as described in Section 2.2) for each system.

inclination. For this reason, in all the plots concerning the mutual inclination (right of Fig. 2 and Fig. 3) a central value with error-bars is drawn on the $y$ coordinate. In Fig. 3 , we show the results for the systems HD 143761 and HD 40307.

We can summarise the results provided by our implementation of the Kolmogorov's normalisation scheme as follows: the systems HD 141399, HD 143761 and HD 40307 are stable in the KAM sense, for mutual inclinations up to $18^{\circ}, 10^{\circ}$ and $15^{\circ}$, respectively. In this context, if we would have taken into account the magnifying factor $1 / \sin i_{j}$ for the mass of the $j$-th planet, we expect that the previous maximal mutual inclinations would be slightly lower, except in the extreme case in which $i_{j}$ are close to zero. Indeed, the main impact of considering larger masses would be increasing the size of the correcting terms of order two in the masses with respect to those of order one in the secular Hamiltonian $H^{(\mathrm{sec})}$.
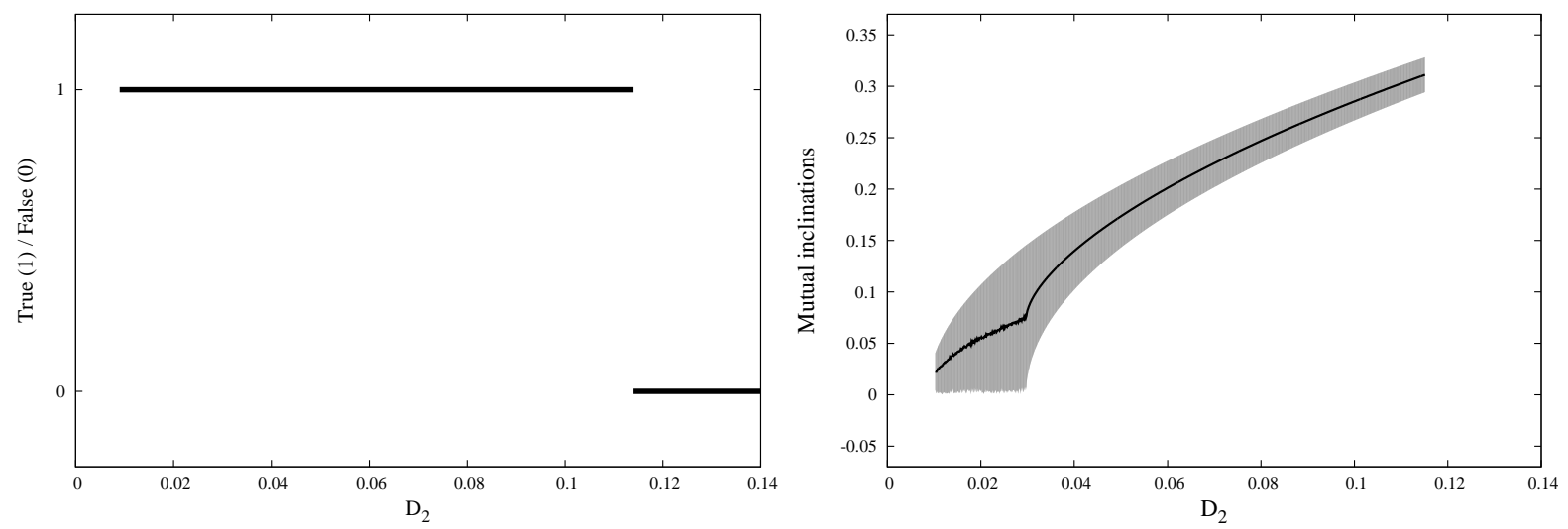

Figure 2: Results relative to HD 141399. On the left, the True/False output regarding the convergence of the algorithm. On the right, the range of values of the mutual inclination (in radians), where the thick line represent the mean value of the inclinations interval. Both the plots are drawn as functions of the parameter $D_{2}$. 

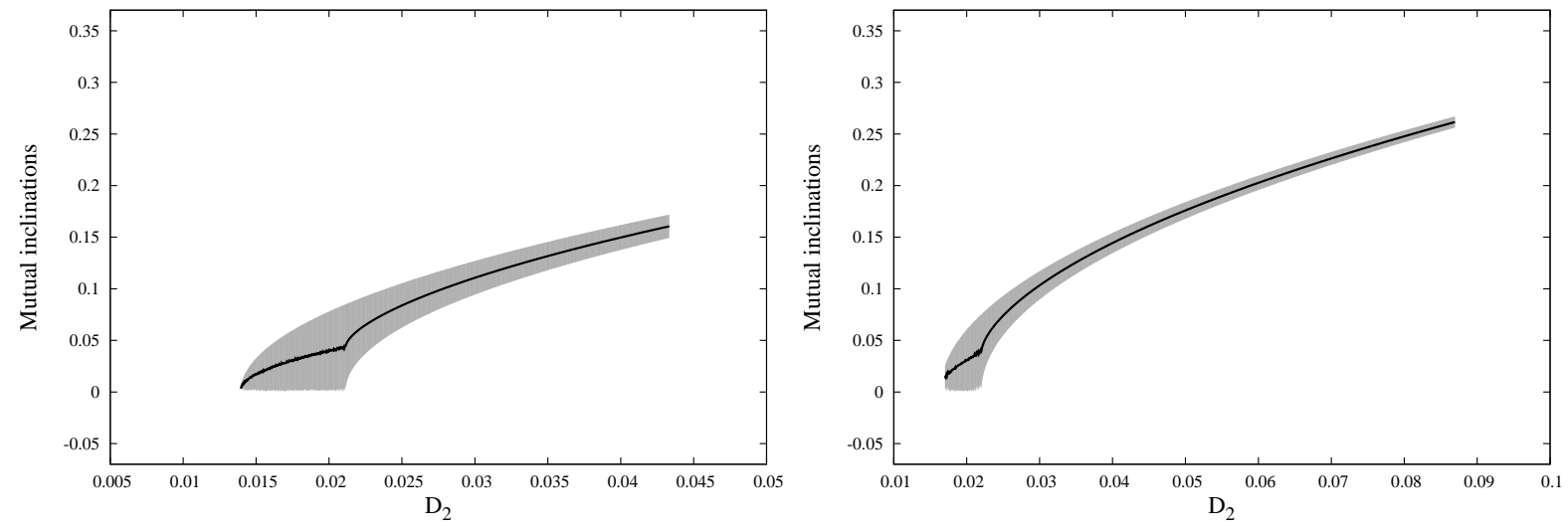

Figure 3: Plots of the mutual inclination as function of the parameter $D_{2}$. On the left, the results relative to HD 143761. On the right, those for HD 40307.

\section{Conclusions and perspectives}

Up to our knowledge, this is the first application of KAM theory to extrasolar planetary systems. As it is discussed in the previous sections, actually we have not applied a statement of the KAM theorem. Instead, we have exploited a keystone of the proof, i.e., the study of the convergence of the generating functions. In this respect we can say that our approach is computer aided: the norms of some of the initial generating functions are evaluated after having explicitly calculated their expansions, instead of being analytically estimated. The eventual convergent character of the constructing algorithm in its entirety is inferred by the behaviour of said norms. Our results should legitimately be included in the list of the applications of KAM theory to realistic physical models (see, e.g., [5, 6, 9]). In fact, for what concerns the tori that are invariant with respect to the secular Hamiltonian and characterized by the complete circulation of the arguments of the pericenters, the values of the mutual inclinations for which the Lidov-Kozai resonant region takes place can be considered as a natural upper limit3. In extrasolar systems such a critical value of the mutual inclinations is usually located at about $40^{\circ}$ (see [24]). Therefore, for the three systems here considered, our results about the stability in the KAM sense cover a set of values whose extension ranges between $25 \%$ and $50 \%$ of the maximal one.

We shall now point out the weaknesses of our approach. Our constructing algorithm does not work when the eccentricities of the planets are not small. In fact, the procedure has generated divergent series when it has been applied to the systems HD 109271, HD 155358 and HD 4732; in all of them there is at least one of the planets whose eccentricity is between 0.1 and 0.25 . Thus, it seems that our approach is limited to systems

\footnotetext{
${ }^{3}$ In the Laplace plane frame, the region of the Lidov-Kozai resonance is characterized by the libration of the argument of the pericenter of the inner planet (see [25]). The implicit adoption of such a frame has been essential in order to perform the reduction of the angular momentum sketched in Sec. 2.1. Therefore, the comparison between our results and those for that resonant region is valid because also our Hamiltonian model is written in the secular canonical coordinates with respect to the Laplace plane.
} 
with planetary eccentricities $<0.1$. Since we are able to produce results for small inclinations of the major planets of the systems, the ideal situation is very similar to that of our Solar System. This is not surprising, since the whole approach has been adapted from the one described in [26], which in turn has been tailored to the Jovian planets. In particular, the series expansion of the three-body planetary Hamiltonian is in power series of some coordinates and parameters that are of the same order of the eccentricities and the inclinations.

A natural goal for the future would be to remove the limitations affecting the approach described in this paper. We think that some of them are intrinsic in the definition of stability that we assumed. Actually, since the beginning we postulated that the motions of the major planets are quasi-periodic and their orbits lie on KAM tori constructed with expansions in small eccentricities and inclinations. Such a prescription is extremely strict. In our opinion, any substantial improvement of the method will be based on a clever weakening of the requirements. This should be done by identifying a suitable integrable approximation of the secular dynamics that can be shown to be convergent even for large eccentricities. In the very different context of the orbits of the Trojan bodies, this change of attitude has been shown to produce substantial enhancements (see [30, 31]). In future works, we plan to extend this kind of ideas to the problem of determining values of the inclinations consistent with (a suitable type of) stability.

\section{Acknowledgements}

During the early stages of preparation of this work, U.L. has been financially supported by the project "DEXTEROUS - Uncovering Excellence 2014" of the University of Rome "Tor Vergata".

Every author has no conflicts of interest to declare.

\section{References}

[1] V.I. Arnold: Proof of a theorem of A. N. Kolmogorov on the invariance of quasiperiodic motions under small perturbations of the Hamiltonian, Usp. Mat. Nauk, 18, 13 (1963); Russ. Math. Surv., 18, 9 (1963).

[2] C. Beaugé, S. Ferraz-Mello and T.A. Michtchenko: Multi-planet extrasolar systems detection and dynamics, Research in Astron. and Astroph., 12, 1044-1080 (2012).

[3] L. Biasco, L. Chierchia and E. Valdinoci: N-dimensional elliptic invariant tori for the planar $(N+1)$-body problem, SIAM Journal on Mathematical Analysis, 37, n. 5, 1560-1588 (2006).

[4] R.P. Butler, J.T. Wright, G.W. Marcy, D.A. Fischer, S.S. Vogt, C.G. Tinney, H.R.A. Jones, B.D. Carter, J.A. Johnson, C. McCarthy and A.J. Penny: Catalog of Nearby Exoplanets, Astroph. Jour., 646, 505-522 (2006). 
[5] A. Celletti: Construction of librational invariant tori in the spin-orbit problem, J. of Applied Math. and Physics (ZAMP), 45, 61-80 (1994).

[6] A. Celletti and L. Chierchia: KAM stability and Celestial Mechanics, Memoirs of AMS, 187, n. 878 (2007).

[7] A. Celletti, A. Giorgilli and U. Locatelli: Improved estimates on the existence of invariant tori for Hamiltonian systems, Nonlinearity, 13, 397-412 (2000).

[8] S. Ferraz-Mello: The convergence domain of the Laplacian expansion of the disturbing function, CeMDA, 58, 37-52 (1994).

[9] F. Gabern, A. Jorba and U. Locatelli: On the construction of the Kolmogorov normal form for the Trojan asteroids, Nonlinearity, 18, 1705-1734 (2005).

[10] A. Giorgilli, U. Locatelli and M. Sansottera: On the convergence of an algorithm constructing the normal form for lower dimensional elliptic tori in planetary systems, Cel. Mech. \& Dyn. Astr., 119, 397-424 (2014).

[11] A. Giorgilli, U. Locatelli and M. Sansottera: Secular dynamics of a planar model of the Sun-Jupiter-Saturn-Uranus system; effective stability in the light of Kolmogorov and Nekhoroshev theories, Regular and Chaotic Dynamics, 22, 54-77 (2017).

[12] A. Giorgilli and M. Sansottera: Methods of algebraic manipulation in perturbation theory, in "Chaos, Diffusion and Non-integrability in Hamiltonian Systems - Applications to Astronomy", Proceedings of the Third La Plata International School on Astronomy and Geophysics, P.M. Cincotta, C.M. Giordano and C. Efthymiopoulos eds., Universidad Nacional de La Plata and Asociación Argentina de Astronomía Publishers, La Plata (2012).

[13] A.N. Kolmogorov: Preservation of conditionally periodic movements with small change in the Hamilton function, Dokl. Akad. Nauk SSSR, 98, 527 (1954). Engl. transl. in: Los Alamos Scientific Laboratory translation LA-TR-71-67; reprinted in: Lecture Notes in Physics, 93.

[14] J. Laskar: Systèmes de variables et éléments, in Benest, D. and Froeschlé, C. (eds.): Les Méthodes modernes de la Mécanique Céleste, 63-87, Editions Frontières (1989).

[15] J. Laskar: A numerical experiment on the chaotic behaviour of the solar system, Nature, 338, 237-238 (1989).

[16] J. Laskar: The chaotic motion of the solar system - A numerical estimate of the size of the chaotic zones, Icarus, 88, 266-291 (1990).

[17] J. Laskar: Large Scale Chaos and Marginal Stability in the Solar System, Cel. Mech. \& Dyn. Astr., 64, 115-162 (1996). 
[18] J. Laskar: Frequency Map analysis and quasi periodic decompositions, in Benest, D., Froeschlé, C. and Lega E. (eds.): Hamiltonian systems and Fourier analysis, Taylor and Francis (2003).

[19] J. Laskar and A.C.M. Correia: HD 60532, a planetary system in a 3:1 mean motion resonance, Astron. \& Astroph., 496, L5-L8 (2009).

[20] J. Laskar and M. Gastineau: Existence of collisional trajectories of Mercury, Mars and Venus with the Earth, Nature, 459, 817-819 (2009).

[21] J. Laskar and A.C. Petit: AMD-stability and the classification of planetary systems, Astron. \& Astroph., 605, A72 (2017).

[22] A.S. Libert and J. Henrard: Analytical study of the proximity of exoplanetary systems to mean-motion resonances, Astronomy and Astrophysics, 461, 759-763 (2007).

[23] A.-S. Libert and M. Sansottera: On the extension of the Laplace-Lagrange secular theory to order two in the masses for extrasolar systems, Celest. Mech. Dyn. Astr., 117, 149-168 (2013).

[24] A.-S. Libert and K. Tsiganis: Kozai resonance in extrasolar systems, Astron. \& Astroph., 493, 677-686 (2009).

[25] M.L. Lidov: The evolution of orbits of artificial satellites of planets under the action of gravitational perturbations of external bodies, Plan. \& Sp. Science, 9, 719-759 (1962).

[26] U. Locatelli and A. Giorgilli: Invariant tori in the secular motions of the three-body planetary systems, Cel. Mech. \& Dyn. Astr., 78, 47-74 (2000).

[27] U. Locatelli and A. Giorgilli: Invariant tori in the Sun-Jupiter-Saturn system, DCDS-B, 7, 377-398 (2007).

[28] A. Morbidelli and A. Giorgilli: Superexponential stability of KAM tori, J. Stat. Phys., 78, 1607-1617 (1995).

[29] J. Moser: On invariant curves of area-preserving mappings of an annulus, Nachr. Akad. Wiss. Gött,. II Math. Phys. Kl 1962, 1-20 (1962).

[30] R.I. Páez and U. Locatelli: Trojan dynamics well approximated by a new Hamiltonian normal form, MNRAS, 453, 2177-2188 (2015).

[31] R.I. Páez, U. Locatelli and C. Efthymiopoulos: New Hamiltonian expansions adapted to the Trojan problem, Cel. Mech. \& Dyn. Astr., 126, 519-541 (2016).

[32] H. Poincaré: Leçons de Mécanique Céleste professées a la Sorbonne, Tome I, Théorie générale des perturbations planetaires, Gautier-Villars, Paris (1905). 
[33] A.C. Petit, J. Laskar and Boué, G.: $x$ AMD-stability in the presence of first-order mean motion resonances, Astron. \& Astroph., 607, A35 (2017).

[34] P. Robutel: Stability of the Planetary Three-Body Problem - II. KAM Theory and Existence of Quasiperiodic motions, Cel. Mech. \& Dyn. Astr., 62, 219-261 (1995).

[35] M. Sansottera, U. Locatelli and A. Giorgilli: On the stability of the secular evolution of the planar Sun-Jupiter-Saturn-Uranus system, Math. Comp. Sim., 88, 1-14 (2013).

[36] J.N. Winn and D.C. Fabrycky: The Occurrence and Architecture of Exoplanetary Systems, Ann. Rev. of Astron. and Astroph., 53, 409-447 (2015). 